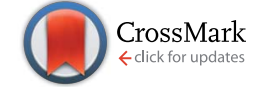

Cite this: RSC Adv., 2015, 5, 12511

Received 18th October 2014 Accepted 8th January 2015

DOI: $10.1039 / c 4 r a 12676 a$

www.rsc.org/advances

\section{A simple low-cost method to enhance luminescence and fluorescence signals in PDMS- based microfluidic devices $\uparrow$}

\author{
Klaus Eyer, Katharina Root, Tom Robinson and Petra S. Dittrich $\ddagger^{*}$ \\ We present a simple and low-cost method to increase the signal intensity originating from poly(dimethyl) \\ siloxane (PDMS) based microfluidic devices by modifying the optical properties of the polymer. The \\ addition of titanium dioxide results in an opaque, white composite with enhanced light reflection \\ properties. The composite can be used to increase the collected light from fluorescent and luminescent \\ moieties inside microfluidic channels by up to 11-fold, as well as reducing the background signal in \\ luminescence studies. Both of these effects increase sensitivity and lower detection limits in analytical \\ setups based on PDMS microfluidic devices. Furthermore, the production of the composite material is \\ simple, low-cost and can be implemented in standard PDMS device fabrication.
}

\section{Introduction}

When optimizing the performance of any analytical setup, sensitivity is an important parameter. In many cases the aim is to ensure that the limit of detection is as low as possible. In the case of fluorescence, several features can be optimized to enhance the performance of the system. ${ }^{1}$ An increase in sensitivity can be achieved by (i) increasing the excitation light intensity, which can result in higher bleaching rates, (ii) improved optical filters and detection instrumentation, or (iii) the use of fluorophores with high quantum yields and/or molar extinction coefficients. Nowadays, even single molecule fluorescence detection can be achieved using photostable fluorophores, ${ }^{2-5}$ but this relies on expensive, non-routine instrumentation.

Integrating luminescence assays into microfluidic systems is even more challenging due to the low amount of molecules available to emit light in such small volumes. Again, sensitivity can be increased with the use of better detection systems or by using luminescent derivatives with higher quantum efficiencies. Many new derivatives have been synthesized over the past decades that have higher intensities than their natural occurring moieties. For example, in the case of coelenterazine that is used in combination with aequorin, newer derivatives can have up to 500 times the intensity of the natural compound. ${ }^{6}$ Also, derivatives with enhanced intensities have been presented for

Department of Chemistry and Applied Biosciences, ETH Zurich, CH-8093 Zurich, Switzerland. E-mail: petra.dittrich@bsse.ethz.ch; Fax: +41 4463212 92; Tel: +41 446336893

† Electronic supplementary information (ESI) available. See DOI: 10.1039/c4ra12676a

\$ Current address: ETH Zurich, Department of Biosystems Science and Engineering, Switzerland. the enzyme luciferase. ${ }^{7-10}$ Although these molecules increase light output and can be used to increase sensitivity, the range of applications is limited. Another way of increasing the sensitivity is to increase the light collection efficiency of the system. As the molecules emit the photons isotropically (see Fig. 1a), only a fraction of light is collected by the objective (depending on the numerical aperture (NA), e.g. even for a high NA 1.4, this is only $\sim 30 \%$ ). Improvements can be made by including mirrored surfaces adjacent to the sample, but such structures are difficult to integrate into microfluidic devices. ${ }^{\mathbf{1 1 - 1 4}}$

Here, we show a simple and low-cost method to increase the signal intensity from fluorescent or luminescent samples by adding titanium dioxide $\left(\mathrm{TiO}_{2}\right)$ particles into poly(dimethyl) siloxane (PDMS) based microfluidic devices (Fig. 1a and 2a). Incorporated particles have been used before to tailor the properties of the PDMS substrate, e.g. to reduce background fluorescence by the addition of carbon-black particles, ${ }^{15}$ to directly integrate long pass filters into diagnostic devices, ${ }^{16}$ to create flexible membranes with magnetic properties ${ }^{17,18}$ or adding zirconate-titanate particles to achieve a composite with vibrational damping properties. ${ }^{19} \mathrm{TiO}_{2}$ has further been used in microfluidics as a coating in electrophoresis applications where it allowed the analysis of amino acids. ${ }^{20}$ Combinations of $\mathrm{TiO}_{2}$ and PDMS have also been used to create a composite with controllable wettability properties, ${ }^{21}$ that can guide fluids inside microfluidic channels. ${ }^{22}$ Here, we take advantage of the fact that $\mathrm{TiO}_{2}$ is able to reflect visible light efficiently and suspend it within PDMS to increase the light collection from microfluidic channels. The channels are covered with a transparent glass slide so that excitation and detection can be performed through the glass without any attenuation of the emitted and reflected light, respectively. 




Fig. 1 Signal enhancement through addition of titanium dioxide. (a) Vertical cross section through the microfluidic chip that consists of PDMS with suspended titanium dioxide and a transparent glass slide. Light emitted from a point source (e.g. a fluorescent molecule) inside the microfluidic channel radiates in all directions. Only light that travels towards the objective is collected and contributes to the signal (left). If the channel walls are reflective due to the implementation of $\mathrm{TiO}_{2}$, light propagating towards the channel walls will be reflected diffusively towards the objective and recorded by the detector additionally to the directly detected fluorescence light. As a result, the signal is enhanced (right). (b) Photographs of $4 \mathrm{~mm}$ thick PDMS pieces with added $\mathrm{TiO}_{2}$. An increase in the concentration of added $\mathrm{TiO}_{2}(\mathrm{w} / \mathrm{w} \%)$ results in an increase in white colorization and opacity of the PDMS.

\section{Materials and methods}

\section{Reagents}

SU8 and developer were obtained from Microchem (Newton, MA, USA). Poly(dimethylsiloxane) (PDMS, Sylgard 184 elastomer kit) was purchased from Dow Corning (Midland, MI, USA). $1 H, 1 H, 2 H, 2 H$-Perfluorodecyl-dimethylchlorosilane and resorufin were procured from ABCR (Karlsruhe, Germany), 2-(4-(2hydroxyethyl)piperazin-1-yl)ethanesulfonic acid (HEPES), fluorescein sodium salt, 4-methylumbelliferone (4-MU), horseradish peroxidase (HRP), sodium carbonate anhydrous and $\mathrm{TiO}_{2}$ nanopowder (powder B) were obtained from Sigma-Aldrich (Buchs, Switzerland). $\mathrm{TiO}_{2}$ powder (powder A) was obtained from Fluka. Dimethylsulfoxide (DMSO) was purchased from Chemie Brunschwig AG (Basel, Switzerland). Hydrogen peroxide 30\% was obtained from MERCK (Zug, Switzerland). Luminol was purchased from TCI (Eschborn, Germany). 8Hydroxy-1,3,6-pyrenetrisulfonic acid trisodium salt (HPTS) was obtained from Acros Organics (Geel, Belgium). Calcein sodium salt was obtained from Fisher Chemicals (Reinach, Switzerland).

\section{Chip fabrication}

Master molds were fabricated by photolithography as previously described. ${ }^{23}$ For channel design, we used a straight channel with one inlet and outlet, a width of $300 \mu \mathrm{m}$ and a height of 70 $\mu \mathrm{m}$. Microfluidic devices were prepared using the Sylgard 184 elastomer kit. The two components were mixed in a ratio of $10: 1$. Before addition, $\mathrm{TiO}_{2}$ powders were sieved to remove large aggregates. Afterwards, the $\mathrm{TiO}_{2}$ was added to the PDMS in various concentrations $(0,0.9,2.7,6,15.4$ or $21.4 \% \mathrm{w} / \mathrm{w})$. The suspension was thoroughly mixed, degassed for half an hour and subsequently poured onto the master mold to a height of 4 $\mathrm{mm}$. Due to the presence of large aggregates, the last $5 \mathrm{~g}$ of the mixture was discarded. The assembly was placed at $80^{\circ} \mathrm{C}$ for 2 hours to cure. After curing, the elastomer was peeled from the wafer and cut into $24 \mathrm{~mm}$ by $24 \mathrm{~mm}$ size devices. Access holes for fluidic connections were punched with a biopsy puncher (1.5 mm diameter, Miltex, York, PA, USA), and a reservoir made of a pipette tip was attached using semi-cured PDMS. The device was exposed to $80{ }^{\circ} \mathrm{C}$ for 1 hour. To close the channels, the PDMS part was bonded to a glass coverslip after plasma activation for 45 seconds at $18 \mathrm{~W}$ and 0.75 mbar (Harrick Plasma Cleaner PDC-32G, Ithaca, NY, USA). The final assembly was placed at $100{ }^{\circ} \mathrm{C}$ for 10 minutes to ensure a stable bond.

\section{Fluorescence and luminescence experiments}

For fluorescence and luminescence measurements, the chip was mounted on an inverted microscope (IX70, Olympus, Japan) equipped with a $10 \times$ objective (NA 0.3) and a CCD camera (EHD imaging $\mathrm{GmbH}$, Damme, Germany). Fluids were added to the reservoir and drawn through the channels using a syringe pump (neMESYS Cetoni, Germany). The fluorophores (dissolved in HEPES $10 \mathrm{mM} \mathrm{pH} \mathrm{7.4)} \mathrm{were} \mathrm{excited} \mathrm{by} \mathrm{a} \mathrm{mercury} \mathrm{arc} \mathrm{lamp,} \mathrm{and}$ the following filters and camera settings were used: fluorescein, HPTS and calcein excitation at $470 / 40 \mathrm{~nm}$, emission above 500 $\mathrm{nm}$ ( $2 \mathrm{~ms}$ exposure time, no gain), resorufin excitation at 535/50 $\mathrm{nm}$, emission above $604 \mathrm{~nm}$ (100 ms exposure time, no gain) and $4-\mathrm{MU}$ excitation at $360 / 40 \mathrm{~nm}$, emission at $460 / 50 \mathrm{~nm}(200$ $\mathrm{ms}$ exposure time, no gain). For luminescence studies, a $6 \mathrm{mM}$ solution of luminol was prepared in $0.5 \mathrm{M}$ sodium carbonate buffer $\mathrm{pH}$ 7.4. To this solution, $4 \%(\mathrm{v} / \mathrm{v})$ of hydrogen peroxide was added. The solution was filtered and introduced on-chip using the syringe pump $\left(2 \mu \mathrm{l} \mathrm{min}{ }^{-1}\right)$. A second syringe was filled with a solution of $50 \mathrm{U} \mathrm{ml}^{-1}$ horseradish peroxidase (HRP) in the same carbonate buffer with added $5 \mu \mathrm{M}$ fluorescein (for focusing) to introduce the luminol reaction catalyst (HRP) to the chip $\left(2 \mu \mathrm{min}^{-1}\right)$. No filters were used in the experiments, and luminescence was monitored using an EMCCD camera (iXon Ultra, Andor, Belfast, UK, 20 gain, $1 \mathrm{~s}$ exposure time, 30 accumulations).

\section{Transmission and reflection measurements}

For transmission measurements, the PDMS layers were mounted onto the inverted microscope. Bright field images (light from a halogen lamp from the top, CCD camera as detector) were obtained using a $10 \times$ objective and a CCD camera. The intensity of the light and camera settings were set to be constant. Reflection measurements were obtained by using the light of the mercury lamp and optical filters for wavelength selection. Light exposure and detection was done from the same side using the same objective. A mirror was used to measure the value of total reflection $(\sim 100 \%)$ and find the optimal camera settings. The PDMS- $\mathrm{TiO}_{2}$ composite chips were afterwards mounted onto the microscope and the images were taken for 
a)

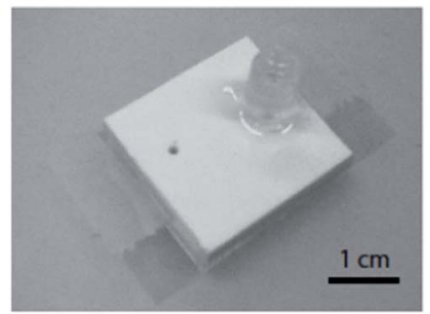

b)

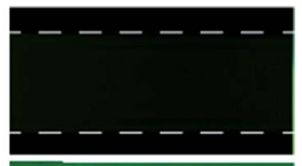

c)

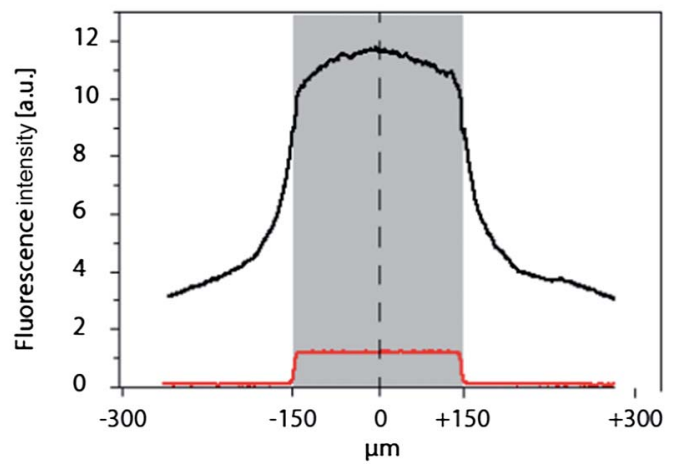

Fig. 2 (a) Photograph of the microfluidic device. To finalize the microfluidic device, the PDMS- $\mathrm{TiO}_{2}$ composite is mounted on a glass slide. The reservoir is used to apply different fluids through the straight channel (not visible in the picture), and the pump is connected to the outlet of the chip. Scale bar $1 \mathrm{~cm}$. (b) In these micrographs, $10 \mu \mathrm{M}$ fluorescein is flushed through the microchannel. The upper micrograph shows a channel inside a chip made from pure PDMS, whereas the lower one contains $12 \%(\mathrm{w} / \mathrm{w}) \mathrm{TiO}_{2}$. Both micrographs are scaled to the same intensities, showing that the signal obtained using the composite device is much higher. Scale bar $150 \mu \mathrm{m}$. (c) Comparison of fluorescence intensities shown in (b). The intensities are measured orthogonal to the channel propagation (channel shown as grey area). Red represents the chip without added $\mathrm{TiO}_{2}$, whereas black represents the chip with $12 \%(\mathrm{w} / \mathrm{w}) \mathrm{TiO}_{2}$. The data reveals an increase in signal intensity as well as the diffuse reflection of fluorescence light visible in the signal measured outside of the channel (i.e. the loss of spatial resolution). Background fluorescence of modified PDMS approaches the same value as unmodified at a distance of approximately $2 \mathrm{~mm}$ (data not shown).

the different wavelengths. A PDMS chip without $\mathrm{TiO}_{2}$ was used to correct the data for PDMS autofluorescence.

\section{Dynamic light scattering measurements}

For dynamic light scattering measurements, the particles were suspended in deionized water. A particle suspension was created by placing the samples in an ultrasonic bath for 10 minutes and by thorough vortexing, both performed shortly before the experiments. Aggregates and larger particles were removed before the measurements by filtration (CellTrics 10 $\mu \mathrm{m}$, Partec). Measurements were conducted with a Zetasizer 3000 (Malvern Instruments) according to the manual.

\section{Results and discussion}

The fabrication of the microfluidic device made of the PDMS$\mathrm{TiO}_{2}$ composite is basically the same as for routine PDMS-based soft lithography. The powder can be suspended in the mixture of PDMS curing agent and prepolymer. Thorough mixing of the components is required, followed by a degassing step. If present, very large aggregates sink during this degassing process to the bottom, and remained in the mixing dish when the composite was poured on a master mold afterwards. We found that none of the used concentrations of $\mathrm{TiO}_{2}$ altered the properties of the composite with respect to flexibility, curing time, the device fabrication or wettability. Likewise, the surface topology of the final PDMS device seems to be unaltered, which we confirmed by taking SEM images (Fig. S1-S3†). Additionally, plasma bonding to glass was successful for all concentrations. With increasing percentage of $\mathrm{TiO}_{2}$, the PDMS appeared more white and opaque and, the light transmittance decreased accordingly (Fig. 1b, Table 1).

First experiments with the composite were done using a chip with suspended $12 \% \mathrm{w} / \mathrm{w} \mathrm{TiO}_{2}$ (Fig. 2a). To investigate the influence of the $\mathrm{TiO}_{2}$ on the signal intensity, we used $10 \mu \mathrm{M}$ concentration of fluorescein dissolved in HEPES $(10 \mathrm{mM}, \mathrm{pH}$ 7.4). Compared to non-altered PDMS, an increase in the signal intensity of around ten-fold was recorded in the channel (Fig. 2b). This enhancement can be attributed to two effects: (i) the reflection of fluorescence light back towards and into the objective and (ii) the reflection of the non-absorbed excitation light back into the sample. There was also an increase in signal adjacent to the channel due to partial reflection of the emitted light from the composite (Fig. 2c).

Next, we measured the collected fluorescence intensities with varying amounts of $\mathrm{TiO}_{2}$ using the following fluorophores: 4-MU $\left(\lambda_{\max }\right.$ emission $\left.=445 \mathrm{~nm}\right)$, fluorescein, HPTS, calcein (all with $\lambda_{\max }$ emission $\left.\approx 514 \mathrm{~nm}\right)$ and resorufin $\left(\lambda_{\max }\right.$ emission $=$ $570 \mathrm{~nm}$ ) (Fig. 3). For all of the tested fluorophore solutions, the signal intensity increased when $\mathrm{TiO}_{2}$ was added (Fig. 3a). For higher $\mathrm{TiO}_{2}$ concentrations the curves flattened indicating saturation of the effect. However, the increase was different for the three tested fluorophores, and we suppose that the different emission wavelengths of the fluorophores determine the maximum signal increase (see also discussion below). For the blue-fluorescent $4-\mathrm{MU}$, the increase in signal intensity was maximal 3.2-fold. However, for the fluorophores with emission maxima in the green and yellow region of the spectrum (fluorescein, HPTS, calcein and resorufin), the increase in the signal was stronger (between 8- and 11.5-fold (Fig. 3b)). It should be noted that the small increase in signal for the buffer solutions is the contribution of the reflected light that can reach the detector due to incomplete blocking of the excitation light by the optical filters.

The addition of $\mathrm{TiO}_{2}$ is particularly useful for luminescence studies, where no excitation light source is required. This was demonstrated using the HRP catalyzed reaction of luminol that is commonly used in bioanalytical assays (Fig. 3c). We could

Table 1 Amount of transmitted white light through a $5 \mathrm{~mm}$ thick PDMS layer with increasing amounts of suspended $\mathrm{TiO}_{2}$ powder $\mathrm{A}$

\begin{tabular}{llllllll}
\hline $\mathrm{TiO}_{2}$ concentration w/w\% & 0 & 0.9 & 2.7 & 6 & 12 & 15.4 & 21.4 \\
Transmission\% & 100 & 1.00 & 0.73 & 0.45 & 0.11 & 0.05 & 0.04
\end{tabular}


a)
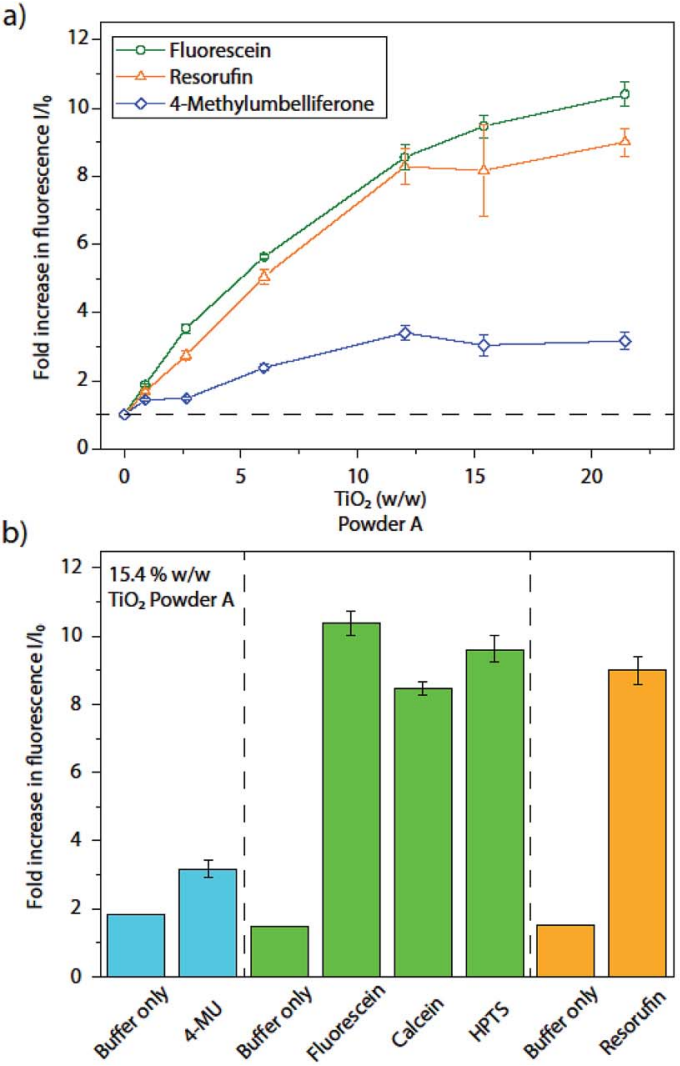

c)

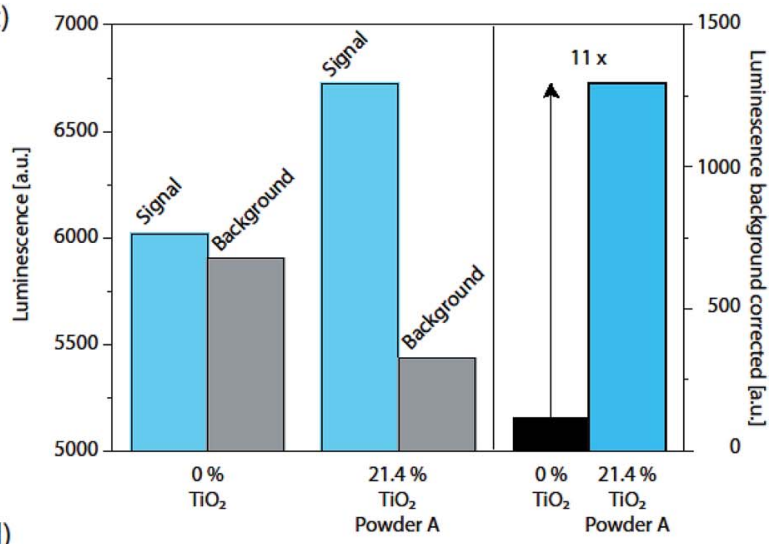

d)

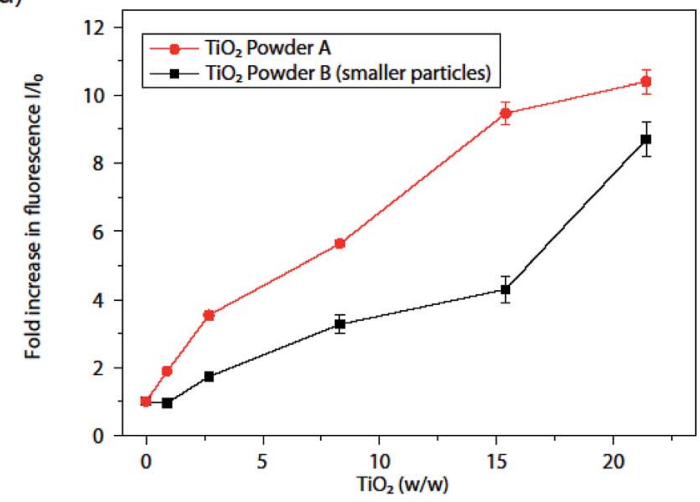

Fig. 3 Results from fluorescence and luminescence experiments. (a) Increase in 4-MU, fluorescein and resorufin (2 $\mu \mathrm{M}, 10 \mu \mathrm{M}, 50 \mu \mathrm{M})$ fluorescence measured in separate devices $(N=3, n=9)$. In each case the fluorescence increases with increasing concentration of suspended TiO powder, although the enhancement is different for each fluorophore. A concentration dependent effect of the increase was not visible in the investigated concentration range. (b) Dependence of the enhancement on the emission wavelength of the fluorophore, all measured using a device with added $15.4 \%$ of $\mathrm{TiO}_{2}$. (c) Luminescence studies with the $\mathrm{TiO}_{2}$ chip. The 11 -fold increase is a result of the reflection of luminescence back into the objective but also due to the reduction of background light. For more information, please refer to the text. (d) To investigate the influence of $\mathrm{TiO}_{2}$ particle size, devices were prepared with two different powders (A and $\mathrm{B}$, different particle sizes) and the increase of fluorescence was measured using $50 \mu \mathrm{M}$ fluorescein. While the suspension of powder B (smaller particle diameter) provided also an increase in signal with increasing concentrations, the signal was always higher for powder $A(N=3)$. This finding is coherent with the theory of light reflection that is dependent on the particle size, please refer to the text for more information. All error bars depict SE values.

observe an increase in signal that can be explained by the scattering properties of $\mathrm{TiO}_{2}$ similar to the observations made in the fluorescence studies. At the same time, the background signal was reduced since the ambient light is blocked by the opaque $\mathrm{TiO}_{2}$-PDMS composite and not recorded on the detector (see also Table 1 for transmittance results). The impact of background reduction is also visible in fluorescence experiments, but is more prominent in luminescence assays due to the longer exposure times and the absence of optical filters. The combination of both effects results in an 11-fold increase in signal after background correction, making the system very suitable and beneficial for luminescence-based studies.

In addition, we investigated the effect of two different particle sizes, purchased from two different suppliers (powder A from Sigma-Aldrich; powder B from Fluka). The size distributions of the particles are measured by DLS (in aqueous solution) and for both powders we found smaller particles (small particle diameter of powder A: $270 \pm 50 \mathrm{~nm}$; powder B: $100 \pm 50 \mathrm{~nm}$ ), next to aggregates with larger diameters of $500-800 \mathrm{~nm}$ (Fig. S4†). Fig. 3d depicts the signal increase for a fluorescein solution with increasing concentrations. The use of powder A results in a stronger increase than powder B for all used concentrations. This result was further affirmed by the relative reflection profiles of the two composites at different wavelengths (Table 2). Hence, the powder A with larger particles is superior for the herein described applications.

In our experiments we emphasized the practical use of the composite materials for fabricating microfluidic devices. The underlying effect for the signal enhancement with $\mathrm{PDMS}^{-\mathrm{TiO}_{2}}$ composite is diffusive reflection (scattering) of light. With the condition used here, i.e. light in the visible region, we can assume that the scattering process at the titanium dioxide particles occurs at the transition stage between Rayleigh and more predominantly, Mie scattering. According to Rayleigh theory, we can expect stronger scattering for larger particles as we have seen in Fig. 3d. However, with increasing Mie scattering there is an optimum size of the particles depending on the wavelength, i.e. the maximum scattering can be expected, when the particle size is half of the scattered wavelength, and for very large particles (diameter $\gg$ wavelength) the strength of 
Table 2 Amount of reflected light from $5 \mathrm{~mm}$ thick PDMS layers with $21.4 \% \mathrm{TiO}_{2}$ powder $\mathrm{A}$ or $\mathrm{B}$, at the indicated wavelength range as determined by the optical filter. Due to the autofluorescence of PDMS,19 the data was normalized using PDMS without $\mathrm{TiO}_{2}$

\begin{tabular}{lllllll}
\hline Wavelength range & $340-380$ & $385-425$ & $420-490$ & $510-560$ & $520-600$ & $600-660$ \\
$\begin{array}{l}\text { Relative reflection\% } \\
\mathrm{TiO}_{2} \text { powder A }\end{array}$ & 57.1 & 52.7 & 57.4 & 66.1 & 67.4 & 69.2 \\
$\begin{array}{l}\text { Relative reflection\% } \\
\mathrm{TiO}_{2} \text { powder B }\end{array}$ & 50.2 & 43.8 & 54.6 & 59.6 & 61.8 & 64.6 \\
\end{tabular}

scattering is independent of the scattered light wavelength. ${ }^{\mathbf{2 4 2 5}}$ We used intermediate sizes of particles $(270 \pm 30 \mathrm{~nm})$ compared to the scattered wavelength, which explains the larger signal increase of the green- and red-fluorescent dyes with fluorescence light $>500 \mathrm{~nm}$.

As a recommendation for optimum signal increase, the particle size should be adapted to the choice of the fluorophore and its emission spectrum. In addition, we believe that any other white pigment would have a similar effect, however, titanium dioxide particles with their large refractive index $(n>2.55$, depending on the mineral form) compared to PDMS ( $n \sim 1.4$ in the visible wavelength region) are particularly suitable. They are also widely used in food, cosmetic and coating industries and therefore available in various particle sizes at low cost. ${ }^{26}$ At this point it should be noted that due to the photocatalytic nature of $\mathrm{TiO}_{2}$ nanoparticles, chemical reactions might occur that could have an unwanted effect on an assay. ${ }^{27}$ The degree of this effect would strongly depend on the chemical structure of the investigated analyte as well as the wavelength and intensity of the incident light. The photocatalytic properties of $\mathrm{TiO}_{2}$ are prominent in the UV range, and can therefore be circumvented by using appropriate light sources, filters and wavelengths for the analysis of the biological or chemical sample.

In summary, we have demonstrated a simple way to increase the amount of collected fluorescence and luminescence in PDMS-based microfluidic channels. By using the $\mathrm{PDMS}^{-\mathrm{TiO}_{2}}$ composite material for the chip fabrication, optical signals emitted from the sample are efficiently collected and recorded by the detector. We demonstrated a 3.6 to 11-times increase in sensitivity of fluorescence and luminescence based assays, respectively. The composite material can be used in a wide variety of applications where maximizing the efficiency of light collection is essential.

\section{Acknowledgements}

The use of the clean room facility FIRST is gratefully acknowledged. We thank P. E. Verboket for the fabrication of the master molds and the SEM images. The work was funded by the European Research Council (ERC Starting Grant no. 203428, “n $\mu$ LIPIDS”, P.S.D.), and Merck Serono (E.K.).

\section{References}

1 J. C. Waters, J. Cell Biol., 2009, 185, 1135-1148.

2 W. E. Moerner and D. P. Fromm, Rev. Sci. Instrum., 2003, 74, $3597-3619$.
3 P. Anger, P. Bharadwaj and L. Novotny, Phys. Rev. Lett., 2006, 96, 113002.

4 A. Jain, R. Liu, Y. K. Xiang and T. Ha, Nat. Protoc., 2012, 7, 445-452.

5 P. S. Dittrich and A. Manz, Anal. Bioanal. Chem., 2005, 382, 1771-1782.

6 O. Shimomura, B. Musicki, Y. Kishi and S. Inouye, Cell Calcium, 1993, 14, 373-378.

7 R. Shinde, J. Perkins and C. H. Contag, Biochemistry, 2006, 45, 11103-11112.

8 K. M. Solntsev, S. P. Laptenok and P. Naumov, J. Am. Chem. Soc., 2012, 134, 16452-16455.

9 G. R. Reddy, W. C. Thompson and S. C. Miller, J. Am. Chem. Soc., 2010, 132, 13586-13587.

10 W. Miska and R. Geiger, J. Biolumin. Chemilumin., 1989, 4, 119-128.

11 K. T. Seale, R. S. Reiserer, D. A. Markov, I. A. Ges, C. Wright, C. Janetopoulos and J. P. Wikswo, J. Microsc., 2008, 232, 1-6.

12 E. Le Moal, E. Fort, S. Lévêque-Fort, F. P. Cordelières, M.-P. Fontaine-Aupart and C. Ricolleau, Biophys. J., 2007, 92, 2150-2161.

13 H. Choumane, N. Ha, C. Nelep, A. Chardon, G. O. Reymond, C. Goutel, G. Cerovic, F. Vallet and C. Weisbuch, Appl. Phys. Lett., 2005, 87, 031102.

14 E. Le Moal, E. Fort, S. Lévêque-Fort, A. J. Hideyuki Murata, F. P. Cordelières, M.-P. Fontaine-Aupart and C. Ricolleau, J. Biomed. Opt., 2007, 12, 024030.

15 W. Hellmich, D. Greif, C. Pelargus, D. Anselmetti and A. Ros, J. Chromatogr. A, 2006, 20, 195-200.

16 O. Hofmann, X. Wang, A. Cornwell, S. Beecher, A. Raja, D. D. C. Bradley, A. J. deMello and J. C. deMello, Lab Chip, 2006, 6, 981-987.

17 F. N. Pirmoradi, L. Cheng and M. Chiao, J. Micromech. Microeng., 2010, 20, 015032.

18 W. Wang, Z. Yao, J. C. Chen and J. Fang, J. Micromech. Microeng., 2004, 14, 1321.

19 S. K. Sharma, H. Gaur, M. Kulkarni, G. Patil, B. Bhattacharya and A. Sharma, Compos. Sci. Technol., 2013, 77, 42-51.

20 J.-D. Qiu, L. Wang, R.-P. Liang and J.-W. Wang, Electrophoresis, 2009, 30, 3472-3479.

21 K. Nakata, H. Kimura, M. Sakai, T. Ochiai, H. Sakai, T. Murakami, M. Abe and A. Fujishima, ACS Appl. Mater. Interfaces, 2010, 2, 2485-2488.

22 F. Villafiorita-Monteleone, E. Mele, G. Caputo, F. Spano, S. Girardo, P. D. Cozzoli, D. Pisignano, R. Cingolani, D. Fragouli and A. Athanassiou, RSC Adv., 2012, 2, 95439550 . 
23 P. Kuhn, K. Eyer, S. Allner, D. Lombardi and P. S. Dittrich, Anal. Chem., 2011, 83, 8877-8885.

24 W. M. Hayes, D. R. Lide and T. J. Bruno, in Handbook of Chemistry and Physics, Taylor and Francis Ltd, 95th edn, 2014.
25 J. M. Oyarzun, Pigment Processing, Vinzentz Verlag, Hannover 2000.

26 A. Weir, P. Westerhoff, L. Fabricius, K. Hristovski and N. von Goetz, Environ. Sci. Technol., 2012, 46, 2242-2250.

27 A. Fujishima, T. N. Rao and D. A. Tryk, J. Photochem. Photobiol., A, 2000, 1, 1-21. 